\title{
Maladies congénitales du squelette de la membrane érythrocytaire
}

La connaissance de la structure des protéines du squelette de la membrane érythrocytaire a permis de définir les supports moléculaires de certaines maladies congénitales du globule rouge et d'en démontrer l'hétérogénéité. Les anomalies les mieux individualisées intéressent la spectrine et la protéine 4. 1, dont les méthodes d'étude ont été bien définies.

\section{Pierre Boivin}

Directeur de l'unité Inserm U I60, chef du service d'hématologie de l'hôpital Beaujon.

\section{REFERENCES}

I. Cohen CM. The molccular organization of the red cell membrane skcleton. Semin Hematol 1983 ; 20: $141-58$.

2. Marchesi VT. The red cell membrane skeleton: recent progress. Blood I983; 6r : 1-11.

3. Boivin P. Interactions moléculaires des protéines de la membrane et déformabilité érythrocytaire. Pathol Biol I984; 32 : 717-35.

4. Laemmli UK. Cleavage of the structural proteins during the assmbly of the head of bacterophage $\mathrm{T}_{4}$. Nature $1970 ; 227: 680-5$.

5. Cleveland DW, Fischer SG, Kirschner MW Laemmli UK. Peptide mapping by limited proteolysis in sodium dodecyl sulf ate and analysis by gel electrophoresis. $7 \mathrm{Biol}$ Chem 1977 252 : $1102-6$.

6. Speicher OW, Morrow JS, Knowles WJ Marchesi VT. Identification of proteolytically resistant domains of human erythrocyte spectrin. Proc Natl Acad Sci USA 1980; 77 : 5673-7.

7. Morrow JS, Speicher DW, Knowles WJ, Hsu $\mathrm{J}$, Marchesi V. Identification of functional domains of human erythrocytes spectrin. Proc Natl Acad Sci USA 1980; 77 : 6592-6.

\section{ADRESSE}

P. Boivin, Inserm Uı6o, Hôpital Beaujon, 92 I I 8

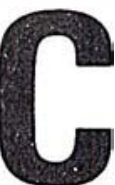
omme les autres membranes cellulaires, celle du globule rouge est formée d'une double couche de phospholipides opposés par leurs groupements hydrophobes et stabilisés par du cholestérol libre, et de protéines parmi lesquelles on distingue des protéines dites intégrales ou transmembranaires parce qu'elles traversent de part en part la double couche de lipides et des protéines dites extrinsèques parce qu'elles sont en dehors de celle-ci vers l'intérieur ou l'extérieur du globule rouge $[\mathrm{I}]$. Celles situées à la face interne de la membrane constituent essentiellement le squelette membranaire. Celui-ci est un filet, un grillage qui tapisse toute la surface interne de la bicouche lipidique en mailles irrégulières bien objectivées par la microscopie à haute résolution. Trois protéines essentielles entrent dans la constitution de ce squelette : la spectrine, l'actine et la protéine 4.1 .

L a spectrine est une protéine allongée dont la molécule de base est un hétérodimère formé d'une chaîne $\alpha$ de MR 240 ooo et d'une chaine $\beta$ un peu plus légère de MR 220000 . Les deux chaines enroulées l'une sur l'autre forment des fibres flexibles et déformables d'environ roo nanomètres de long. Deux dimères s'attachent l'un à l'autre par leur extrémité proximale, dite tête, pour former des tétramères et des oligo- mères de plus haut degré [2] qui constituent les formes normales de la spectrine in vivo. Les deux chaines $\alpha$ et $\beta$ sont impliquées dans la tétramérisation. Cette interaction entre deux molécules de dimère est une propriété fondamentale de la spectrine. Par leur extrémité distale, des tétramères s'attachent les uns aux autres pour former les " nœuds " des mailles du filet; cette liaison se fait par l'intermédiaire de l'actine, forme particulière paucipolymérisée de $\beta$ actine; l'interaction entre spectrine et actine est considérablement facilitée par la présence de la protéine 4.1 elle-même formée de deux composants $4.1 a$ et 4.16 de masse moléculaire respective 82000 et 78 ooo. Le squelette membranaire est attaché au reste de la membrane par une protéine d'ancrage, l'ankyrine qui se lie d'une part à la chaîne $\beta$ de la spectrine, d'autre part au segment cytoplasmique de la principale protéine transmembranaire nommée protéine 3. D'autres ancrages semblent se faire par l'intermédiaire d'une interaction entre la protéine 4.1 et une des glycoprotéines membranaires, la glycophorine C, glycoconnectine ou glycoprotéine $\beta$ et par des interactions moléculaires entre les protéines du squelette et les lipides membranaires [3].

Nous ne traiterons ici que des anomalies moléculaires des protéines membranaires qui semblent primiti- 
ves, à l'exclusion de celles liées à la présence d'un déficit enzymatique cytosolique ou d'une hémoglobine pathologique.

\section{Méthodes d'étude}

La mise en évidence et l'identification d'une anomalie moléculaire ou d'une interaction anormale entre les protéines du squelette membranaire nécessite la mise en œuvre de multiples épreuves de laboratoire. Celles-ci doivent être pratiquées sur les prélèvements sanguins frais afin d'éviter les artéfacts.

L'aspect morphologique des globules rouges peut être apprécié sur les frottis sanguins mais l'examen à l'état frais d'une suspension de globules rouges fixés à la glutaraldehyde rend mieux compte de la forme des érythrocytes et du pourcentage des formes anormales. L'étude de la déformabilité au moyen de l'ektacytomètre, en faisant varier l'osmolalité, permet de définir une courbe de l'indice ektacytométrique caractéristique des divers types d'anomalies membranaires congénitales. Une telle épreuve est particulièrement utile dans les études génétiques.

La sensibilité thermique des érythrocytes est étudiée en incubant des globules rouges pendant io à 60 minutes à des températures variant de 43 à $49^{\circ} \mathrm{C}$. Les GR incubés sont examinés au microscope à la recherche de bourgeonnements et de fragmentations membranaires et/ou à l'ektacytomètre.

L'analyse globale des protéines membranaires après dissolution des ghosts dans le SDS est faite par électrophorèse sur gel de polyacrylamide SDS. La technique de Laemmli [4] permet une excellente séparation des protéines en particulier des $4.1 a$ et $4.1 b$. Les gels sont colorés au bleu de Coomassie pour l'identification des peptides, à l'acide périodique-Schiff ou à l'argent pour la mise en évidence des glycoprotéines. Lorsqu'un constituant protéique de mobilité anormale est mis en évidence par l'électrophorèse, il peut être difficile de savoir quel constituant normal il remplace ou de quel peptide il provient. Les méthodes immunologiques sont ici nécessaires : la technique de l'immunoblot avec les immun-sérums mono ou polyclonaux spécifiques permet l'identification du constituant anormal.

L'étude fonctionnelle des interactions entre molécules protéiques est un temps fondamental de l'identification des lésions membranaires.

(a) Interactions entre dimères de spectrine. L'interaction entre dimères in vivo est reflétée par l'état de la spectrine extraite à faible force ionique et $4^{\circ} \mathrm{C}: 85 \%$ au moins de la spectrine sont sous forme de tétramères, I $5 \%$ au plus sous forme dimérique. L'extraction à $37^{\circ} \mathrm{C}$ à faible force ionique fournit essentiellement des dimères de spectrine. Incubés à $30^{\circ} \mathrm{C}$ en conditions isotoniques les dimères se transforment en tétramères jusqu'à atteindre un point d'équilibre thermodynamique; la pratique de cette transformation in vitro permet de définir une constante d'association dimèredimère qui pour la spectrine normale est dans notre laboratoire de $6( \pm 0,4) \times 10^{5} \mathrm{M}^{-1}$. Dans toutes ces épreuves les quantités respectives de dimères et de tétramères peuvent être appréciées après séparation des deux espèces soit par gel filtration soit par sédimentation en gradient de sucrose, soit par électrophorèse sur gel de polyacrylamide non dénaturant.

(b) Étude fonctionnelle de la protéine 4.1. En présence de protéine 4.1, une forte interaction entre spectrine et actine apparait, aboutissant à la formation d'un complexe ternaire stable actine-protéine 4.1-spectrine. Cette propriété de la protéine 4.1 peut être étudiée in vitro en observant l'incorporation de spectrine dimérique dans des complexes protéiques de haut poids moléculaire composés de spectrine et d'actine. Ainsi, il faut ajouter I $55 \mu \mathrm{g}$ de protéine 4.1 à I $\mathrm{mg}$ de spectrine brute pour observer une disparition de $50 \%$ du dimère de spectrine présent dans cette extraction. Les études les plus fines et les plus riches en enseignement ont été menées en utilisant de la protéine 4.1 marquée à $l^{125} \mathrm{I}$ et de la spectrine elle aussi marquée à $\mathrm{l}^{125}$ I ou par phosphorylation. La mise en contact de quantités variables de ces protéines et la séparation du complexe formé soit par gradient de sédimentation soit par méthodes immunologiques permet de déterminer les quantités respectives de protéines qui fixent l'une et l'autre. Une autre technique très élégante utilise une colonne d'affinité de sepharose-protéine 4.1 sur laquelle on fait passer de la spectrine purifiée; l'élution étant faite par un gradient de force ionique. Un défaut de fixation à une protéine 4.1 normale évoque l'existence d'une anomalie moléculaire de la chaîne $\beta$ de la spectrine.

(c) L'interaction entre spectrine et ankyrine est étudiée par la fixation de spectrine purifiée marquée par un radio-isotope soit ${ }^{125} \mathrm{I}$ soit ${ }^{32} \mathrm{P}$ sur des vésicules retournées déplétées en spectrine-actine. On peut ainsi calculer au moyen du diagramme de Schatchard le nombre de sites de fixation et leur affinité. Cette méthode de même que celle d'étude de l'interaction ankyrinebande 3 est de réalisation difficile et de résultats souvent incertains.

Études structurales : Une digestion protéolytique limitée peut être pratiquée soit sur les chaînes $\alpha$ et $\beta$ isolées soit sur les dimères ou tétramères de spectrine. La méthode de Cleveland [5] permet d'étudier les chaines $\alpha$ et $\beta$ isolées à partir de très faibles quantités de protéines membranaires. Cependant, la méthode de Speicher et al. [6] de protéolyse de spectrine est plus généralement employée pour permettre les comparaisons des résultats d'un laboratoire à l'autre. Cette méthode a permis de définir des domaines relativement résistants à la protéolyse correspondant à des peptides volumineux que l'on peut séparer par électrophorèse en gel d'acrylamide en une ou en deux dimensions par combinaison d'électrophorèse et d'électrofocalisation : cinq domaines dénommés $\alpha \mathrm{I}$ à $\alpha \mathrm{V}$ pour la chaîne $\alpha$, quatre pour la chaîne $\beta$ ( $\beta$ I à $\beta$ IV) [7]. Dans le cadre des malades héréditaires, les domaines jusqu'ici concernés sont surtout le peptide de $80 \mathrm{~K}$ ou domaine I de la chaîne 
$\alpha(\alpha \mathrm{I})$ et le peptide $28 \mathrm{~K}$ de la chaîne $\beta(\beta$ I) porteur de l'extrémité $\mathrm{COOH}$ terminale et des sites de phosphorylation de cette chaîne.

\section{Anomalies moléculaires de la spectrine}

Elliptocytoses constitutionnelles avec anomalies de la chaîne $\alpha$ de la spectrine.

Les elliptocytoses dites de type I fréquentes chez les sujets de race noire sont rencontrées principalement à l'état hétérozygote chez l'adulte où elles ne s'accompagagnent que d'une hémoluse modérée ou nulle. Chez les nouveaux-nés hétérozygotes elles sont parfois cause d'une hémolyse intense avec poikylocytose et schizocytose, hémolyse dont l'évolution est favorable au cours de la première année de la vie au bout de laquelle le tableau clinique et hématologique redevient progressivement celui de l'elliptocytose hétérozygote de l'adulte que porte l'un des parents. A l'état homozygote, le tableau est celui d'une hémolyse intense de l'enfant avec poïkilocytose et pycnocytose. Les anomalies de la spectrine sont soupçonnées sur les données suivantes :

(a) lors des extractions à $4^{\circ} \mathrm{C}$ à faible force ionique, le pourcentage de dimères (normalement inférieur à I $5 \%$ ) est constamment augmenté, généralement entre 20 et plus de $50 \%[8,9]$;

(b) la constante d'association des dimères in vitro $\left(n=6 \times 10^{5} \mathrm{M}^{-1}\right.$ $\pm 0,4)$ est diminuée (de I à $\left.3 \times 10^{5} \mathrm{M}^{-1}\right)$

(c) l'épreuve de stabilité thermique montre un abaissement de la température de dénaturation inférieur aux $49^{\circ} \mathrm{C}$ normaux et qui peut atteindre 43 à $47^{\circ} \mathrm{C}$ surtout chez les homozygotes [9].

L'affirmation des anomalies est apportée par la digestion protéolytique ménagée et l'établissement des cartes peptidiques [10]. Dans les elliptocytoses de type I les anomalies jusqu'ici décrites atteignent le segment I de la chaîne $\alpha$ de la spectrine qui est représenté par un peptide de 80000 de poids moléculaire. Ce peptide est constamment diminué, d'environ $50 \%$ chez les hétérozygotes, de sa totalité chez les homozygotes. Des peptides anormaux apparaissent, de plus faibles poids moléculaires, qui répondent à trois variants jusqu'ici isolés : - un variant de $74 \mathrm{KD}$ donnant lieu, selon la nomenclature actuelle à un phénotype $\left(\mathrm{Sp} \mathrm{D} \alpha^{\mathrm{l} / 74}\right)(\mathrm{SpD}=$ spectrine dimère, $\alpha$ I répond au segment impliqué, 74 au PM du peptide). Le génotype hétérozygote est alors $\left(\mathrm{SpD} \alpha^{1 / 74}\right)$ $-(\mathrm{SpD})$ et $\left(\mathrm{SpD} \alpha^{1 / 74}\right)$ - $\left(\mathrm{Sp} D \alpha^{1 / 74}\right)$ chez les homozygotes. - un variant de $46 \mathrm{KD}$ (souvent accompagné d'un peptide de $22 \mathrm{KD})$ répondant aux phénotypes $\left(\mathrm{Sp} \mathrm{D} \alpha^{1 / 46}\right)$ et aux génotypes respectifs $\left(\mathrm{Sp} \mathrm{D} \alpha^{1 / 46}\right)-(\mathrm{SpD})$ et $\left(\mathrm{Sp} \mathrm{D} \alpha^{1 / 46}\right)-\left(\mathrm{Sp} D \alpha^{1 / 46}\right)$ chez les hétéro et homozygotes. - un variant de $6_{5} \mathrm{KD}$, probablement le plus fréquent [ $\mathrm{II}$ ] avec le génotype $\left(\mathrm{Sp} \mathrm{D} \alpha^{1 / 65}\right)$ et les phénotypes $\left(\mathrm{Sp} \mathrm{D} \alpha^{1 / 65}\right)-(\mathrm{SpD})$ et $\left(\mathrm{SpD} \alpha^{1 / 65}\right)$ $-\left(\mathrm{SpD} \alpha^{1 / 65}\right)$. Les températures de dénaturation sont normales chez les hétérozygotes porteurs du peptide 74 KD. Elles sont modérément abaissées $\left(47^{\circ} \mathrm{C}\right)$ chez les sujets hétérozygotes porteurs des peptides 46 et $65 \mathrm{KD}$. Elles sont constamment et fortement abaissées chez les enfants hétérozygotes avec poïkilocytose (autour de $45^{\circ} \mathrm{C}$ ) et chez les homozygotes, quel que soit le variant (autour de $45^{\circ} \mathrm{C}$ ). Que l'enzyme protéolytique coupe la molécule à des emplacements différents de la normale prouve, soit que les liaisons peptidiques coupées ont une position anormale par mutation structurale, soit que la conformation anormale de la molécule expose à l'action de l'enzyme des sites normalement masqués. Dans les deux hypothèses une anomalie moléculaire est présente. A côté de ces variants accompagnant une elliptocytose, d'autres variants ont été dépistés chez des sujets de race noire en l'absence de toute manifestation pathologique. Ils sont situés sur les domaines $\alpha_{2}$ et $\alpha_{3}$ de la chaîne $\alpha$ de la spectrine et constituent un simple polymorphisme génétique. Ils peuvent coexister avec les variants $\alpha_{1}$ de l'elliptocytose $[10,12]$

Elliptocytoses avec anomalies de la chaîne $\beta$ de la spectrine (a) Un premier type bien défini al. Molecular from patients with hereditary pyropoikilocytosis. f Clin Invest 1983; 71 : 1867-77. 


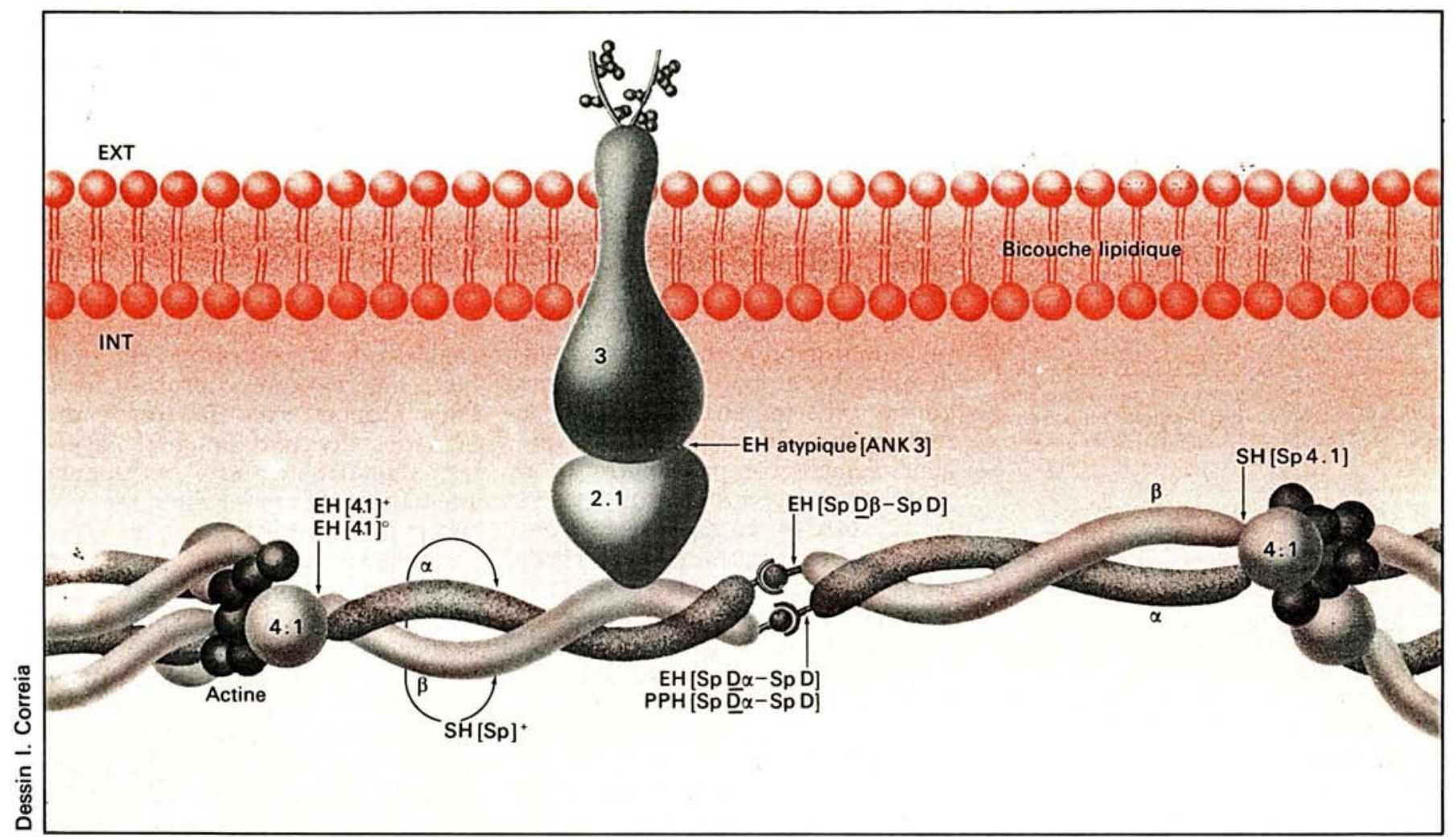

Représentation schématique du cytosquelette avec la localisation des anomalies moléculaires connues au cours des elliptocytoses héréditaires (EH), de la pyropoïkilocytose héréditaire (PPH) et de la sphérocytose héréditaire (SH). $\mathrm{EH}[4.1]^{+}$: déficit partiel en protéine 4.1

$E H[4.1]^{\circ}$ : déficit complet en protéine 4.1

$S H[S p]^{+}$: déficit partiel en spectrine

$S H[S p 4.1]$ : défaut d'intéraction de la spectrine avec la protéine 4. I normale

$E H[A N K 3]$ : défaut d'intéraction entre l'ankyrine et la protéine bande 3 normale in situ.

$E H[S p-D \beta-S p D]$ : défaut d'autoassociation en dimère de spectrine; le dimère pathologique est modifié sur sa chaîne $\beta$

$E H$ et $P P H[S p-D \alpha-S p D]$ : défaut d'autoassociation des dimères de spectrine; le dimère pathologique est modifié sur sa chaîne $\alpha$

répond à une observation unique mais fondamentale dans la mesure où elle comporte la première démonstration formelle d'une anomalie structurale de la molécule de spectrine [13]. Elle concerne une femme et son père, porteurs d'une hémolyse modérée, et d'une elliptocytose avec poïkilocytose. La caractéristique biochimique est l'existence chez les deux sujets d'une chaine $\beta$ de la spectrine anormale, transmise à l'état hétérozygote et représentant $50 \%$ des chaînes $\beta$ totales. L'anomalie structurale consiste en un raccourcissement de la chaîne $\beta$ par absence d'un peptide de 6000 environ de masse moléculaire; l'absence totale de phosphorylation de la chaîne mutée localise sur ce peptide les sites normaux de phosphorylation de la spectrine; le dimère formé de la chaîne $\alpha$ normale et de la chaîne $\beta$ mutée est totalement incapable de se lier à un autre dimère pour former un tétramère apportant la preuve de la nécessité de l'intégrité du segment capital de la chaîne $\beta$ dans le processus de tétramérisation. Bien que non démontré, le mécaniqme génétique pourrait être une délétion d'un segment important d'un gène de structure de la chaîne $\beta$.

(b) Une autre variété d'anomalies de la chaîne $\beta$ est suspectée sur les données de la protéolyse ménagée de la spectrine [14]. Les perturbations n'intéressent pas la chaîne $\alpha$, les deux chaînes de spectrine ont un aspect électrophorétique normal mais il existe un trouble de l'équili- bre dimère-tétramère avec un excès de dimère dans les extractions à $4^{\circ} \mathrm{C}$ et une diminution de la tétramérisation in vitro [ $\mathrm{I}_{5}$ ]. L'anomalie moléculaire correspondante n'est pas identifiée; elle semble intéresser l'extrémité céphalique de la chaîne $\beta$ mais differre certainement de celle décrite dans le chapitre précédent.

Pyropoïkilocytose héréditaire et anomalies de la chaîne $\alpha$ de la spectrine : il s'agit là d'une maladie hémolytique rare observée essentiellement dans les populations noires. Elle est caractérisée chez les propositus par une hémolyse néonatale de la petite enf ance, de haute gravité, nécessitant des transfusions sanguines répétées à court intervalle et obligeant fréquemment à prati- 


\section{REFERENCES}

18. Lawler J, Liu SC, Palck J, Prchal J. Molecular defect of spectrin in heteditary pyropoikilocytosis. Alterations in the trypsinresistant domain involved in spectrin self-association. 3. Clin Invest 1982; 70 : 1019-30.

19. Lawler J, Palek J, Prchal J, Butler WM. Molecular heterogeneity of hereditary pyropoikilocytosis : identification of a second variant of the spectrin $\alpha$-subunit. Blood 1983; 62 : 11 82-9.

20. Greenquist A, Shohet SB, Bernstein SE. Marked reduction of spectrin in hereditary spherocytosis in the common house mouse. Blood 1978; 51 : 1149-55.

21. Goodman SR, Shiffer KA, Casoria LA, Eyster ME. Identification of the molecular defect in the erythrocyte membrane skeleton of some kindreds with hereditary spherocytosis. Blood 1982 ; $60: 772-84$.

22. Wolfe LC, John KM, Falcone JC, Byrne AM, Lux S. A genetic defect in the binding of protein 4.1 to spectrin in a kindred with hereditary spherocytosis. N Engl 7 Med 1982; 307 : 1367-73.

23. Becker PS, Cohen CM, Lux SE. Mild oxidation alters the structure of human erythrocyte spectrin (Abst). Blood 1984; 64 Suppl. 1 : $23 a$.

24. Agre P, Orringer EP, Bennett V. Deficient red-cell spectrin in severe recessively inherited spherocytosis. N Engl 7 Med 1982; 306 : 11 55-61.

25. Féo CS, Fisher S, Piau JP, Grange MJ, Tchernia G. Première observation de l'absence d'une protéine de la membrane érythrocytaire (bande 4. I) dans un cas d'anémie elliptocytaire familiale. Nouv Rev Fr Hematol 1980; 22 : 31525.

26. Garbarz M, Dhermy D, Lecomte MC, et al. A variant of erythrocyte membrane skeletal protein band 4.1 associated with hereditary elliptocytosis. Blood 1985; 64 : 1006-15.

27. Alloisio N, Dorleac E, Delaunay J, Girot R, Galand C, Boivin P. A shortened variant of red cell membrane protein 4. 1. Blood 1982; 60 : 265-7.

28. Smith JE, Moore K, Arens M, Rinderknecht GA, Ledet A. Hereditary elliptocytosis with protein band 4.1 deficiency in the dog. Blood 1983; 61 : 373-7.

29. Hill JS, Sawyer WH, Howlett GJ, Wiley JS. Hereditary spherocytosis in man. Altered binding of cytoskeletal components to the erythrocyte membrane. Biochem 7 1981; 201 : 259-66.

30. Agre P, Orringer EP, Chui DHK, Bennett V. A molecular def ect in two families with hemolytic poikilocytic anemia. Reduction of high affinity membrane binding sites for ankyrin. $\mathcal{J}$ Clin Invest I $981 ; 68: 1566-76$. quer une splénectomie précoce qui améliore constamment l'hémolyse. Sur le plan strictement hématologique, les caractéristiques des hématies sont une grande anisopoikilocytose avec de nombreuses fragmentations globulaires, de microcytes, des hématies bourgeonnantes, quelques ovalocytes et sphérocytes.

Les anomalies biochimiques sont :

(a) une instabilité thermique considérable des globules rouges et des ghosts avec un abaissement de la température de fragmentation qui, généralement comprise entre 45 et $47^{\circ} \mathrm{C}$, peut descendre jusqu'à $43^{\circ} \mathrm{C}$. Cette baisse est en rapport avec une dénaturation thermique anormale de la spectrine et fait partie de la définition même de la maladie;

(b) l'extraction à $4^{\circ} \mathrm{C}$ fournit la spectrine essentiellement sous la forme dimérique qui représente jusqu'à soixante pour cent et plus de l'extrait total [ I6];

(c) l'épreuve de tétramérisation in vitro est caricaturale : seule une faible part de dimère est apte à subir la transformation tétramérique et la constante d'association est effondrée à un chiffre voisin de :

I $\times 10^{5} \mathrm{M}^{-1}\left(n \simeq 6 \pm 0,4 \times 10^{5} \mathrm{M}^{-1}\right)$; (d) l'analyse des cartes peptidiques met en évidence un état hétérozygote caractérisé par une diminution du peptide $80 \mathrm{KD}$ remplacé plus ou moins par un peptide de $\mathrm{Mr} 74 \mathrm{KD}$, $65 \mathrm{KD}$ ou $46 \mathrm{KD}$, anomalies analogues à celles observées dans l'elliptocytose constitutionnelle de type I hétérozygote $[17,18,19]$.

Les données sur la transmission héréditaire montrent que celle-ci est d'apparence récessive et que les malades sont probablement porteurs double-hétérozygotes d'une anomalie moléculaire érythrocytaire. Bien que le nombre d'observations connues soit faible et que le mécanisme de transmission génétique n'ait pas pu être toujours élucidé, il est clair que les deux parents sont souvent normaux cliniquement et selon les données classiques de l'hémogramme. Dans certains cas l'un des parents est porteur d'une elliptocytose constitutionnelle de type I, conf orme à la description ci-dessus et dont la spectrine possède les caractères pathologiques décrits [9]. Même lorsqu'il en est ainsi chez le second géniteur qui apparaît normal, on peut parfois mettre en évidence les anomalies biochimiques de la spectrine : excès de dimère dans l'extraction à $4^{\circ} \mathrm{C}$, défaut d'interaction dimère-dimère in vitro, diminution du fragment peptidique $80 \mathrm{KD}$ et présence d'un variant $74 \mathrm{KD}, 46$ ou $65 \mathrm{KD}$. L'enfant malade parait donc avoir hérité de ses parents des anomalies de la chaine $\alpha$ de la spectrine à l'état double hétérozygote, probablement sans rapport avec la forme des hématies des porteurs hétérozygotes. Mais dans d'autres cas, aucune anomalie fonctionnelle de la spectrine n'est décelée chez les parents. Ce fait négatif peut avoir deux explications hypothétiques : soit que nos méthodes d'analyse de la spectrine soient encore trop sommaires pour permettre le dépistage d'anomalies présentes à l'état hétérozygote et " masquées " par la présence d'une part de spectrine normale, soit que le ou les troubles présents chez les parents intéressent des systèmes d'interactions non encore connus.

Sphérocytose héréditaire : si jusqu'ici aucune anomalie qualitative de protéines membranaires n'avait pu être décrite au cours des sphérocytoses héréditaires à transmission dominante, l'atteinte de la spectrine y était déjà pratiquement certaine sur les arguments suivants :

l'instabilité à l'urée des squelettes des hématies de la sphérocytose héréditaire;

l'impossibilité pour les extraits membranaires (spectrine brute) de stimuler la polymérisation d'actine-G ajoutée au milieu;

l'impossibilité de former un gel en exposant les coquilles à la phosphorylation par la spectrine-kinase purifiée;

l'insuffisance de phosphorylation du composant 2 de la spectrine, inconstante certes mais expliquée non par un déficit des activités protéine-kinasiques mais par un défaut de sites de phosphorylation; la formation expérimentale de sphérocytes par action de substances végétales telles la vinblastine et la colchicine qui dénaturent la spectrine.

En outre un modèle animal de sphé- 


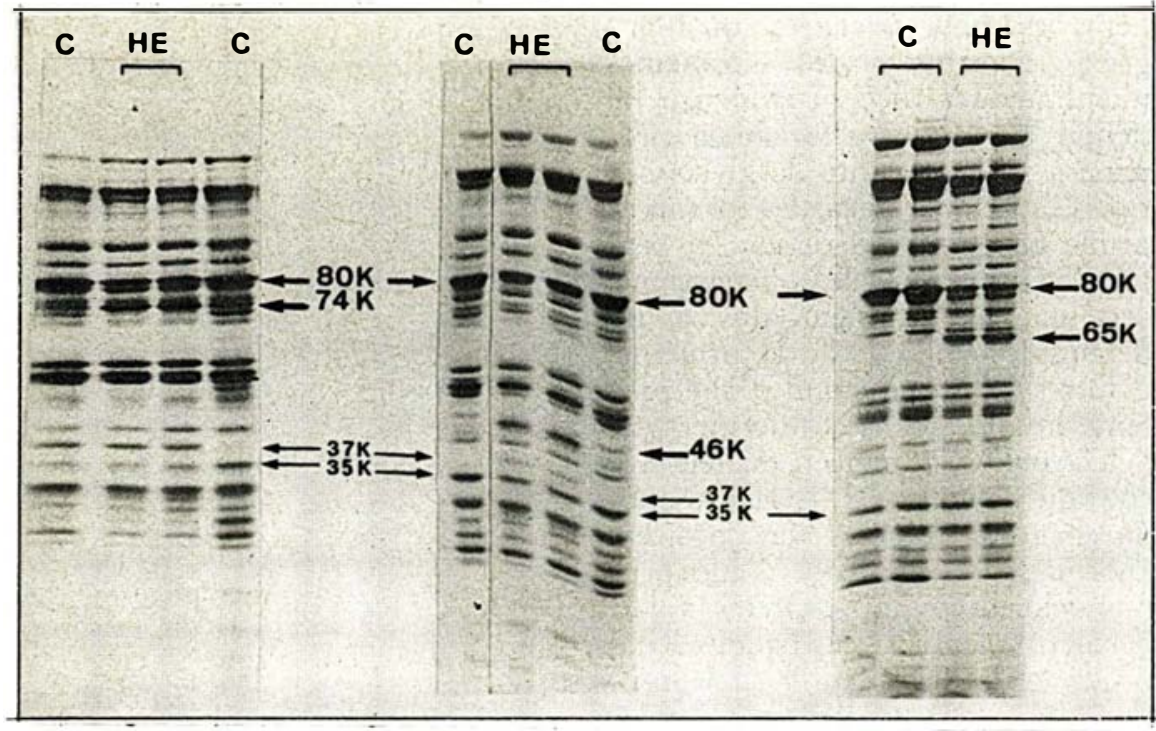

Mise en évidence par électrophorèse unidimensionnelle des peptides de protéolyse ménagée répondant aux trois variants communs du peptide alpha-I de la spectrine chez des sujets atteints d'elliptocytose héréditaire (HE).

Dans ce type d'expérience les peptides sont séparés selon leur taille, ceux de hauts poids moléculaires migrant plus lentement (haut du gel) que ceux de bas poids moléculaires (bas du gel)

$K=$ milliers en unités de poids moléculaire ( $80 K=80000$ daltons)

Par rapport aux témoins $(C)$ on note la diminution du peptide $80 \mathrm{~K}$ et la présence de peptides $74 \mathrm{~K}, 46 \mathrm{~K}$ et $65 \mathrm{~K}$. Les anomalies présentes au niveau des peptides 37-35 $K$ sont dues au polymorphisme génétique du segment alpha-2.

rocytose héréditaire a été décrit chez la souris, caractérisé par une grande instabilité érythrocytaire, la présence de sphérocytes avec bourgeonnements et fragmentation érythrocytaire et par un déficit de spectrine. Plusieurs types en existent où la gravité de l'anémie hémolytique et l'intensité des lésions globulaires sont directement en rapport avec la gravité du déficit en spectrine : plus intense est celui-ci plus grave est l'hémolyse [20].

(a) Sphérocytose héréditaire de type I : chez l'homme, l'affirmation d'une anomalie moléculaire de la spectrine comme responsable de certains cas de sphérocytose héréditaire a été récemment apportée par deux équipes américaines qui ont démontré l'existence d'un trouble des interactions entre la spectrine et la protéine 4.1 [21, 22]. L'anomalie a été trouvée chez environ un tiers des familles de sphérocytose héréditaire typique qui ont été étudiées. L'application des méthodes décrites ci-dessus a montré que tous les malades étaient porteurs de deux types de molécules de spectrine : l'une a toutes les caractéristiques de la spectrine normale; l'autre qui représente environ $40 \%$ de la spectrine totale a pour caractéristique de ne pas se fixer sur la protéine 4. 1, tant sur la colonne de protéine 4. 1 sepharose, que dans les expériences utilisant la protéine 4.1 ${ }^{125} \mathrm{I}$. Aucune autre interaction n'est pertubée: L'anomalie est située semble-t-il sur la chaine $\beta$ à l'extrémité opposée aux sites de fixation de l'ankyrine et de la phosphorylation. La présence de deux types de molécules traduit bien l'état hétérozygote classique de la sphérocytose héréditaire. Il est proposé de dénommer variante I ce type de maladie, bien défini par son anomalie fonctionnelle.

(b) Anomalies des groupes sulfhydriles de la spectrine au cours de la sphérocytose héréditaire : chez près de la moitié des malades atteints de sphérocytose héréditaire, surtout dans les cas atypiques par l'absence de l'une quelconque des caractéristiques habituelles de la maladie, il existe une diminution modérée et globale de la spectrine portant sur les chaînes $\alpha$ et $\beta$ et mise en évidence sur les gels de polycrylamide par l'étude du rapport spectrine/bande 3. Le déficit est en moyenne de l'ordre de $10 \%$ et peut atteindre $30 \%$. Chez les mêmes sujets, la quantité de dimère de spectrine dans les membranes et dans l'extraction à $4^{\circ} \mathrm{C}$ est normale mais la constante d'association dimèredimère in vitro est légèrement diminuée $\left(4\right.$ à $5 \times 10^{5} \mathrm{M}^{-1}$ pour une normale de $\left.6 \times 10^{5} \mathrm{M}^{-1} \pm 0,4\right)$.

La signification de ces anomalies est incertaine. Le rôle des groupes sulfhydriles de la spectrine est probable : en présence de réducteurs, l'épreuve de tétramérisation est normalisée (D. Dhermy et coll. résultats non publiés). De plus, l'oxydation ménagée des groupes SH.d'une spectrine normale reproduit l'anomalie de l'interaction spectrine-protéine 4.1 décrite dans la sphérocytose héréditaire de type I et la réduction secondaire corrige le trouble d'interaction [23]; deux conclusions peuvent en être tirées : d'une part, qu'un des groupements $\mathrm{SH}$ de la spectrine est impliquée dans la sphérocytose héréditaire de type I (mais il n'a pas été démontré que le trouble de ce type de sphérocytose pouvait être reversé par la 
réduction des groupes $\mathrm{SH}$ de la spectrine pathologique); d'autre part, qu'il pouvait être suspecté que certaines au moins des anomalies décrites soient secondaires à des phénomènes d'oxydation des groupes $\mathrm{SH}$ et non dues à une anomalie moléculaire structurelle primitive.

(c) Déficit en spectrine et sphérocytose héréditaire récessive : une maladie différente de la sphérocytose héréditaire classique a été décrite comme une grande microsphérocytose à transmission récessive. Elle est supportée par une diminution de plus de $50 \%$ de la spectrine dans les hématies du propositus; ce déficit porte plus sur la chaîne $\alpha$ que sur la chaîne $\beta$ et n'est pas retrouvée chez les parents. Les techniques immunologiques ne mettent pas en évidence de spectrine ou de produits de dégradation de celle-ci dans les hémolysats. Le mécanisme de ces altérations quantitatives reste incertain et la possibilité d'une protéolyse ne peut pas être totalement exclue [24].

\section{Anomalies de la protéine 4.1}

Elles constituent la seconde classe d'anomalies moléculaires des elliptocytoses constitutionnelles. Jusqu'ici les anomalies de la protéine 4.1 n'ont été recontrées que chez des sujets de race blanche. Le pourcentage d'elliptocytes varie d'un cas à l'autre, de même que l'aspect plus ou moins allongé des érythrocytes. $\mathrm{La}$ transmission est autosomale dominante. Dans les formes homozygotes qui sont jusqu'ici exceptionnelles, existe une anémie hémolytique sévère, améliorée par la splénectomie, accompagnée d'elliptocytose, de poikilocytose, de sphérocytose. Une absence totale de protéine 4.1 est objectivée par l'électrophorèse des protéines membranaires [25]. La protéine de liaison entre la protéine 4.1 et le reste de la membrane qui est la glycophorine $C$ (glycoprotéine $\beta$ ou glycoconnectine) manque elle aussi. Cette absence parait secondaire à celle de la protéine 4.1 . Dans les formes hétérozygotes, l'hémolyse hématies plus ovalaires qu'allongées; l'analyse des protéines membranaires met en évidence un déficit d'environ $50 \%$ de la protéine 4.1 ; la quantité de glycophorine $\mathrm{C}$ y semble normale. Le mécanisme génétique du déficit en protéine 4.1 semble hétérogène; certains cas semblent dus à un défaut de synthèse de la protéine; d'autres à la production d'une protéine instable; dans ces derniers cas l'électrophorèse met en évidence, à côté du déficit en 4.1 , la présence de bandes colorées en position anormale; l'emploi d'anticorps monoclonaux anti-protéine 4.1 permet d'identifier ces bandes à des produits de dégradation de la protéine 4.1 [26]. Ces mêmes études ont permis de reconnaitre également un polymorphisme génétique de la protéine 4.1 dépourvu de tout caractère pathologique [27].

Le rôle du déficit en protéine 4.1 dans l'elliptocytose héréditaire a été discuté; il existe cependant un modèle animal : une lignée de chiens a été reconnue dans laquelle coexistaient une anémie hémolytique avec elliptocytose et un déficit de la protéine 4.1 [28].

\section{Anomalies de l'ancrage du squelette}

Les cas rapportés sont moins démonstratifs et l'authenticité des lésions moléculaires fonctionnelles moins solidement établie que dans les cas précédents.

Dans certaines sphérocytoses héréditaires, une inextractibilité de la spectrine a été rapportée [29]. Dans d'autres, la fixation du complexe spectrine-actine sur les vésicules retournées témoignerait d'une diminution du nombre de sites de fixation; le siège de l'anomalie serait plutôt sur l'ankyrine que sur la spectrine.

Dans deux familles avec elliptocytose, poikilocytose et hyperhémolyse, les vésicules retournées déplétées en ankyrine ne fixaient pas l'ankyrine normalement, alors que l'ankyrine des patients se fixait normalement à des vésicules déplétées provenant d'hématies témoins. Il a été suggéré que l'anomalie primitive était portée par la bande 3 plutôt que par l'ankyrine [30]

\section{Summary}

Progress achieved in recent years in the knowledge of the proteins of the erythrocyte membrane skeleton have enabled scientists to define the molecular basis of certain congenital diseases of the red blood cell and to demonstrate their heterogeneity. The most specific anomalies are related to spectrin and protein 4.1 , for which methods of study have been well-defined and standardized. Several variants of alpha and beta chains of spectrin have been discovered in the course of constitutional elliptocytosis; located on the segments of chains believed to be involved in the formation of spectrin tetramers, their presence inhibits the formation of tetramers and leads to an instability of the skeleton. Identical variants have been observed in hereditary pyropoikilocytosis. An anomaly of spectrin affecting the segment of the beta chain responsible for interacting with protein 4.1 has been described in hereditary spherocytosis. Different types of protein 4.1 deficits have been detected in hereditary elliptocytoses. Anomalies in the attachment of the skeleton have been encountered in different types of congenital hemolytic anemias.

\section{TIRES A PART}

P. Boivin, Inserm Uı6o, Hôpital Beaujon, 92118 Clichy Cedex. 